OPEN ACCESS

Edited by:

Hong Ni,

Children's Hospital of Soochow University, China

Reviewed by: Rosa Marotta,

University of Magna Graecia, Italy Enrico Domenici,

University of Trento, Italy

*Correspondence: Rosanna Weksberg rweksb@sickkids.ca

Specialty section: This article was submitted to

Pediatric Neurology,

a section of the journal

Frontiers in Neurology

Received: 30 September 2020 Accepted: 15 March 2021

Published: 16 April 2021

Citation:

Jangjoo M, Goodman SJ, Choufani S,

Trost B, Scherer SW, Kelley E,

Ayub M, Nicolson R, Georgiades S,

Crosbie J, Schachar R, Anagnostou E, Grunebaum E and Weksberg R (2021)

An Epigenetically Distinct Subset of

Children With Autism Spectrum Disorder Resulting From Differences in Blood Cell Composition

Front. Neurol. 12:612817.

doi: 10.3389/fneur.2021.612817

\section{An Epigenetically Distinct Subset of Children With Autism Spectrum Disorder Resulting From Differences in Blood Cell Composition}

\author{
Maryam Jangjoo ${ }^{1}$, Sarah J. Goodman ${ }^{1}$, Sanaa Choufani ${ }^{1}$, Brett Trost ${ }^{1,2}$, \\ Stephen W. Scherer 1,2,3,4, Elizabeth Kelley ${ }^{5}$, Muhammad Ayub ${ }^{5}$, Rob Nicolson ${ }^{6}$, \\ Stelios Georgiades ${ }^{7}$, Jennifer Crosbie ${ }^{8,9}$, Russell Schachar ${ }^{8,9,10}$, Evdokia Anagnostou ${ }^{11,12}$, \\ Eyal Grunebaum ${ }^{10,13,14}$ and Rosanna Weksberg 1,3,10,12,15* \\ ${ }^{1}$ Genetics and Genome Biology, The Hospital for Sick Children, Toronto, ON, Canada, ${ }^{2}$ The Centre for Applied Genomics, \\ The Hospital for Sick Children, Toronto, ON, Canada, ${ }^{3}$ Department of Molecular Genetics, University of Toronto, Toronto, \\ ON, Canada, ${ }^{4}$ McLaughlin Centre, University of Toronto, Toronto, ON, Canada, ${ }^{5}$ Department of Psychiatry, Queen's \\ University, Kingston, ON, Canada, ${ }^{6}$ Department of Psychiatry, University of Western Ontario, London, ON, Canada, \\ ${ }^{7}$ Department of Psychiatry and Behavioural Neurosciences, Offord Centre for Child Studies, McMaster University, Hamilton, \\ ON, Canada, ${ }^{8}$ Neurosciences and Mental Health Program, The Hospital for Sick Children, Toronto, ON, Canada, \\ ${ }^{9}$ Department of Psychiatry, University of Toronto, Toronto, ON, Canada, ${ }^{10}$ Institute of Medical Science, School of Graduate \\ Studies, University of Toronto, Toronto, ON, Canada, ${ }^{11}$ Holland Bloorview Kids Rehabilitation Hospital, Toronto, ON, Canada, \\ ${ }^{12}$ Department of Pediatrics, University of Toronto, Toronto, ON, Canada, ${ }^{13}$ Division of Immunology and Allergy, The Hospital \\ for Sick Children, Toronto, ON, Canada, ${ }^{14}$ Developmental and Stem Cell Biology Program, The Hospital for Sick Children, \\ Toronto, ON, Canada, ${ }^{15}$ Division of Clinical and Metabolic Genetics, The Hospital for Sick Children, Toronto, ON, Canada
}

Background: Autism spectrum disorder (ASD) is a complex neurodevelopmental disorder that often involves impaired cognition, communication difficulties and restrictive, repetitive behaviors. ASD is extremely heterogeneous both clinically and etiologically, which represents one of the greatest challenges in studying the molecular underpinnings of ASD. While hundreds of ASD-associated genes have been identified that confer varying degrees of risk, no single gene variant accounts for $>1 \%$ of ASD cases. Notably, a large number of ASD-risk genes function as epigenetic regulators, indicating potential epigenetic dysregulation in ASD. As such, we compared genome-wide DNA methylation (DNAm) in the blood of children with ASD $(n=265)$ to samples from age- and sex-matched, neurotypical controls $(n=122)$ using the Illumina Infinium HumanMethylation450 arrays.

Results: While DNAm patterns did not distinctly separate ASD cases from controls, our analysis identified an epigenetically unique subset of ASD cases ( $n=32$ ); these individuals exhibited significant differential methylation from both controls than the remaining ASD cases. The CpG sites at which this subset was differentially methylated mapped to known ASD risk genes that encode proteins of the nervous and immune systems. Moreover, the observed DNAm differences were attributable to altered blood cell composition, i.e., lower granulocyte proportion and granulocyte-to-lymphocyte ratio in the ASD subset, as compared to the remaining ASD cases and controls. This ASD subset did not differ from the rest of the ASD cases in the frequency or type of high-risk genomic variants. 
Conclusion: Within our ASD cohort, we identified a subset of individuals that exhibit differential methylation from both controls and the remaining ASD group tightly associated with shifts in immune cell type proportions. This is an important feature that should be assessed in all epigenetic studies of blood cells in ASD. This finding also builds on past reports of changes in the immune systems of children with ASD, supporting the potential role of altered immunological mechanisms in the complex pathophysiology of ASD. The discovery of significant molecular and immunological features in subgroups of individuals with ASD may allow clinicians to better stratify patients, facilitating personalized interventions and improved outcomes.

Keywords: ASD, DNA methylation, epigenetics, granulocytes, blood cell proportion

\section{INTRODUCTION}

Autism spectrum disorder (ASD) is a heritable and prevalent neurodevelopmental disorder that is usually defined by impairments in cognition, communication and social interaction as well as by restrictive and/or stereotypical repetitive behaviors (1). Despite intense research efforts during the past decade, no definitive biological or clinical markers for ASD have been identified $(2-4)$. This can be partly explained by the highly heterogeneous nature of ASD, both clinically and etiologically, which represents one of the greatest challenges in studying the molecular basis of ASD. The genetic underpinnings of ASD are mainly ascribed to different genetic variants such as rare copy number variations (CNVs), single-nucleotide variants (SNV) and de novo mutations that have been identified in $\sim 10-20 \%$ of individuals with ASD (5-7). While hundreds of ASD-associated genes have been identified that confer varying degrees of risk, no single gene variant accounts for $>1 \%$ of all ASD cases (8-10). Despite its strong genetic component, several lines of evidence suggest that environmental factors and epigenetic mechanisms may contribute to ASD etiology; however, the molecular mechanisms underlying their contributions to the development of ASD are still unclear $(11,12)$. Epigenetic marks, including DNA methylation (DNAm), are involved in the programming of cellular differentiation and development; it is therefore plausible that the dysregulated DNA methylation patterns caused by genetic and/or environmental factors may permanently disrupt biological pathways involved in normal brain development (13).

\footnotetext{
Abbreviations: ADI-R, autism diagnostic interview revised; ADOS, autism diagnostic observation schedule; ANXA1, ANNEXIN A1; ANKRD22, ankyrin repeat domain 22; ASD, Autism spectrum disorder; SB-5, Stanford Binet Intelligence Scales; CHD7, Chromodomain-helicase-DNA-binding protein 7; CHD8, Chromodomain Helicase DNA Binding Protein 8; CNV, Copy number variant; $\mathrm{CpG}$, Cytosine- phosphate- Guanine; CUL3, Cullin 3; DMR, Differentially methylated region; DNAm, DNA methylation; FDR, False Discovery Rate; G/L Ratio, granulocyte/lymphocyte ratio; GO, Gene ontology; MET, MET protooncogene; NK, Natural killer cell; NSD1, Nuclear Receptor Binding SET Domain Protein 1; PCA, Principal component analysis; REB, Research ethics board; SFARI, Simons Foundation Autism Research Initiative; SHANK2, H3,multiple ankyrin repeat domains 2; SNV, single nucleotide variant; VABSII, Vineland Adaptive Behavior Scales; WAS, Wechsler Abbreviated Scale of Intelligence; WISC, Wechsler Intelligence Scale of Children.
}

There is direct evidence from case-control studies showing altered targeted and genome-wide DNAm and histone acetylation in multiple tissues of affected individuals, supporting a role for epigenetic dysregulation in the development of ASD (14-18). Of note, many ASD-risk genes function as epigenetic regulators, i.e., chromatin remodelers, histone modifying enzymes and transcriptional regulators (19-22).

Many epigenetic studies have investigated ASD-associated DNAm signatures in brain tissue, in order to identify epigenetic alterations potentially causative of or mechanistically related to ASD (23-25); however, they are seriously constrained by small sample sizes and the use of autopsy-derived tissue that may be confounded by post-mortem effects on epigenetic marks. Several candidate gene-based studies revealed DNAm alterations at ASD-risk genes such as SHANK3, OXTR, EN2, and MECP2 in multiple brain regions $(15,17,18,26)$. Further, genome-wide screens of DNAm in the brain of individuals with ASD have identified inconsistent differences at a variety of genomic sites; the differentially methylated CpGs were mainly associated with genes enriched in synaptic function and immune response $(16,24)$. Although there is sufficient evidence for immune dysregulation in individuals with ASD, immune-related genes are not among those that contain loss of function variants in next-generation sequencing studies of autistic individuals, further reinforcing the evidence for the involvement of epigenetic mechanisms in the dysregulated immune system detected in brain samples of ASD-affected individuals.

Easily accessible tissues such as blood are often used in epigenetic studies for biomarker discovery in lieu of target tissues that are difficult to access, such as brain. Recent studies have identified specific peripheral blood DNAm signatures for each of 35 neurodevelopmental/ASD syndromes caused by pathogenic variants in genes that encode epigenetic regulators $(27-30)$. There are a very limited number of genome-wide epigenetic studies that examined DNAm changes in peripheral blood of individuals with ASD $(31,32)$. These studies found inconsistent evidence for DNAm alterations associated with ASD, likely due to small sample sizes $(n<100)$ and a specific focus on twin pairs with a lack of extension to the general population. A recent study by Andrews et al. (33) performed a large case-control metaanalysis of blood samples in autistic patients. This study, despite a 
higher sample size $(n=796)$, found no association between ASD and DNAm at genome-wide significance as no single CpG site achieved statistical significance at a Bonferroni correction level. However, they reported seven $\mathrm{CpG}$ sites that achieved suggestive statistical significance for association with ASD with consistent and stronger effects at the same sites among brain samples (33). Of note, these results were obtained from individuals of different ethnic backgrounds which can influence epigenetic changes as a potential confounding factor (34). Therefore, we reduced the ethnic heterogeneity by collecting the majority of samples from the same ethnicity in the present study.

In this study, we overcame previous limitations by investigating genome-wide DNAm in a large ASD $(n=265)$ cohort to identify blood-derived differentially methylated sites, as compared to control subjects. We hypothesized that, given the suggested role of epigenetics in ASD molecular etiology, epigenetic modifications could act as a useful biomarker that may contribute to the underlying etiology of subsets of patients with ASD. Our results demonstrate that DNAm alterations defined an epigenetically distinct subset of ASD cases that differentiate them from other ASD cases and controls. Notably, these observed DNAm differences were significantly associated with shifts in blood cell composition. Gene ontology analysis of the genes overlapping the differentially methylated CpG sites identified functions relevant to known pathophysiological mechanisms underlying ASD such as immune dysfunction, highlighting the biological significance of our DNAm signals.

\section{METHODS}

\section{Research Participants}

Participants of this study were selected from existing ASD cohorts: the Province of Ontario Neurodevelopmental Disorders (POND) Network, the Simons Simplex Collection (SSC), the Autism Speaks MSSNG project and the Genome Diagnostics Laboratory at The Hospital for Sick Children (SickKids). Participants were enrolled in studies approved by the Research Ethics Boards of the respective institutions (Holland Bloorview Kids Rehabilitation Hospital, Toronto; The Hospital for Sick Children, Toronto; McMaster Children's Hospital, Hamilton; Queen's University, Kingston; Western University, London) and informed consent was obtained from participating subjects and/or their parents or guardians.

ASD study cases consisted of individuals aged 1-18 years with a primary clinical diagnosis of ASD of undefined etiology; to that end, we excluded syndromic ASD cases carrying previously identified pathogenic variants with a known effect on DNA methylation, including variants in Chromodomain Helicase DNA Binding Protein 8 (CHD8), Chromodomainhelicase-DNA-binding protein 7 (CHD7), Nuclear Receptor Binding SET Domain Protein 1 (NSD1), and16p11.2 deletions (21, 28, 30). Clinical diagnoses were confirmed using the Autism Diagnostic Interview-Revised (ADI-R) (35) and Autism Diagnostic Observation Schedule (ADOS) (36) or ADOS-2 (37) by clinical staff formally trained on all measures. The neurotypical control samples matched for age- and sex were selected from a collection available in our laboratory, and the
SSC sample. Individuals in the control group were recruited using physician/parental screening questionnaires. The majority of individuals included in this study are of Caucasian descent (Supplementary Table 1). No significant differences were found between the case and the control group in terms of age and sex. The description of the study sample can be found in Table $\mathbf{1}$.

\section{DNAm Array Processing}

DNA samples from whole blood were sodium bisulfite converted for all ASD cases and controls using the Qiagen EZ DNA Methylation kit (Qiagen, Valencia, CA) according to the manufacturer's protocol. The modified genomic DNA was then hybridized to the Illumina Infinium HumanMethylation 450 BeadChip array to interrogate over 485,000 individual CpG sites in the human genome, at The Center for Applied Genomics (TCAG), SickKids Research Institute, Toronto, Canada. The distribution of the samples on the arrays was randomized between cases and controls. Samples were run on arrays in a total of nine batches, with each batch containing ASD cases and controls.

The minfi Bioconductor R package (38) was used to preprocess the array data and generate Beta $[\beta]$-values from the raw intensity measures. Preprocessing included standard quality control metrics in $\min f$, including density plot, median intensity QC plots, and control probe plots. All samples passed quality control as previously described $(28,30)$. Methylation data were then filtered by removing probes exhibiting low detection $p$ value $>0.05$ in more than $25 \%$ of the samples, cross-reactive probes, probes located on sex chromosomes, probes targeting CpG sites within 10 bp of a single-nucleotide polymorphic sites (SNPs) with a minor allele frequency $>1 \%$, probes with raw beta $=0$ or 1 in $>0.25 \%$ of samples, and non-CpG probes; a total of 427,137 probes were retained after filtering based on these criteria for normalization and downstream differential analysis. Normalization with background subtraction was then performed using Illumina control probes. The resulting $\beta$ values represent percent DNAm, ranging from 0 to 1 corresponding to an unmethylated to a fully methylated CpG site.

\section{Differential DNAm Analysis Between ASD Cases and Neurotypical Controls}

Linear regression was performed using the limma package (39) to identify statistically significant differentially methylated CpG sites between all ASD cases $(n=265)$ and controls $(n=122)$, accounting for covariates including age, sex, batch and estimated cell-type proportion. Blood cell type proportions were estimated using Houseman's algorithm and the Bioconductor packages minfi and FlowSorted.Blood.450k (40). Given that these cell type proportions are highly correlated, only monocyte, granulocyte, and natural killer (NK) proportions were included in the regression model (Supplementary Table 1). The remaining cell types were highly correlated with granulocyte proportion $(r>$ $0.6, p$-value $<0.05)$. We computed the false discovery rate (FDR) using the Benjamini-Hochberg method (41). A significant difference in DNAm between ASD cases and controls was called for each $\mathrm{CpG}$ site that met the cutoffs of FDR adjusted 
TABLE 1 | Demographic characteristics for ASD cases and neurotypical controls.

\begin{tabular}{|c|c|c|c|c|}
\hline Sample groups & $\begin{array}{l}\text { Control } \\
(n=122)\end{array}$ & $\begin{array}{l}\text { Full ASD sample } \\
\qquad(n=265)\end{array}$ & $\begin{array}{l}\text { DNAm-based } \\
\text { ASD subset }(n=32)\end{array}$ & $\begin{array}{l}\text { Remaining ASD } \\
\text { sample }(n=233)\end{array}$ \\
\hline Sex & $\mathrm{N}$ & $\mathrm{N}$ & $\mathrm{N}$ & $\mathrm{N}$ \\
\hline Male & 84 & 220 & 27 & 193 \\
\hline Female & 38 & 45 & 5 & 40 \\
\hline \multirow[t]{2}{*}{ Age (years) } & Mean \pm SD & Mean \pm SD & Mean \pm SD & Mean \pm SD \\
\hline & $12.20 \pm 4$ & $8.82 \pm 4$ & $7 \pm 4.50$ & $9.10 \pm 4$ \\
\hline DNA collection site & $\mathrm{N}$ & $\mathrm{N}$ & $\mathrm{N}$ & $N$ \\
\hline TCAG (POND/MSSNG) & 6 & 220 & 26 & 194 \\
\hline SSC & 30 & 43 & 6 & 37 \\
\hline Genome Diagnostics Lab (SickKids) & - & 2 & - & 2 \\
\hline Weksberg Lab (SickKids) & 86 & - & - & - \\
\hline Cell type proportion & Mean \pm SD & Mean \pm SD & Mean \pm SD & Mean \pm SD \\
\hline B cell & $0.10 \pm 0.03$ & $0.11 \pm 0.04$ & $0.16 \pm 0.04$ & $0.11 \pm 0.04$ \\
\hline CD4T & $0.17 \pm 0.04$ & $0.20 \pm 0.06$ & $0.31 \pm 0.06$ & $0.18 \pm 0.05$ \\
\hline CD8T & $0.10 \pm 0.03$ & $0.11 \pm 0.04$ & $0.16 \pm 0.05$ & $0.10 \pm 0.04$ \\
\hline Granulocytes & $0.51 \pm 0.10$ & $0.47 \pm 0.10$ & $0.31 \pm 0.05$ & $0.50 \pm 0.10$ \\
\hline Monocytes & $0.10 \pm 0.02$ & $0.08 \pm 0.02$ & $0.05 \pm 0.02$ & $0.08 \pm 0.02$ \\
\hline NK & $0.05 \pm 0.04$ & $0.04 \pm 0.04$ & $0.02 \pm 0.04$ & $0.04 \pm 0.04$ \\
\hline $\mathrm{G} / \mathrm{L}$ ratio & $1.38 \pm 0.50$ & $1.11 \pm 0.50$ & $0.48 \pm 0.10$ & $1.20 \pm 0.50$ \\
\hline Clinical Measures & & Mean \pm SD & Mean \pm SD & Mean \pm SD \\
\hline $\begin{array}{l}\text { ADI_R: Communication domain verbal } \\
\text { total }\end{array}$ & & $\begin{array}{c}16.52 \pm 4.60 \\
(N=200)\end{array}$ & $\begin{array}{l}16.50 \pm 4.30 \\
(N=22)\end{array}$ & $\begin{array}{c}16.60 \pm 4.60 \\
(N=178)\end{array}$ \\
\hline ADI_R algorithm total scores & & $\begin{array}{l}43.10 \pm 11 \\
(N=110)\end{array}$ & $\begin{array}{l}41 \pm 11 \\
(N=10)\end{array}$ & $\begin{array}{l}43 \pm 11 \\
(N=100)\end{array}$ \\
\hline $\begin{array}{l}\text { ADOS: Communication + Social } \\
\text { Interaction total score }\end{array}$ & & $\begin{array}{c}13.60 \pm 4.60 \\
(N=168)\end{array}$ & $\begin{array}{c}14.30 \pm 4.10 \\
(N=17)\end{array}$ & $\begin{array}{l}13.50 \pm 5 \\
(N=151)\end{array}$ \\
\hline $\begin{array}{l}\text { ADOS: Social Affect total + Restricted } \\
\text { and Repetitive Behavior total score }\end{array}$ & & $\begin{array}{c}16.5 \pm 6.10 \\
(N=52)\end{array}$ & $\begin{array}{l}19 \pm 5.40 \\
(N=9)\end{array}$ & $\begin{array}{l}16.10 \pm 6 \\
(N=43)\end{array}$ \\
\hline $\begin{array}{l}\text { VABS-II: Communication Standard } \\
\text { Score }\end{array}$ & & $\begin{array}{l}78 \pm 16.5 \\
(N=123)\end{array}$ & $\begin{array}{l}80 \pm 16 \\
(N=14)\end{array}$ & $\begin{array}{l}76.50 \pm 18 \\
(N=109)\end{array}$ \\
\hline IQ-Scale (FSIQ score) & & $80 \pm 30(N=129)$ & $\begin{array}{c}80 \pm 30 \\
(N=114)\end{array}$ & $\begin{array}{c}83.60 \pm 27 \\
(N=15)\end{array}$ \\
\hline
\end{tabular}

NK, Natural killer cell; G/L, Granulocyte/Lymphocyte.

$p$-value $<0.01$ and an effect size of 5\% mean methylation difference $(|\Delta \beta|>0.05)$.

\section{Functional and Genomic Enrichment of Differentially Methylated CpG Sites}

For genomic enrichment analysis, the differentially methylated CpG sites were submitted to GREAT 4.0.4 (Genomic Regions Enrichment of Annotations Tool) (42) using a maximum near gene extension of $10 \mathrm{~Kb}$ and a hypergeometric FDR $q$-value $<0.01$ for significance. Enrichment of the gene lists in each Gene Ontology (GO) term was defined against the background set of all probes that remained in the data after minfi probe filtering $(n=427,137)$. Terms with two or more gene hits were reported. We also compared our differentially methylated genes with known SFARI Gene ASD-risk genes (https://gene.sfari.org/) to further understand the biological relevance of our DNAm signal. In addition, we compared the genomic distribution of differentially methylated $\mathrm{CpG}$ sites to the background set of probes for relation to $\mathrm{CpG}$ island and overlapping enhancer region using a hypergeometric test ( $p$-value $<0.05$ ).

\section{Identification of Differentially Methylated Regions}

To identify significantly differentially methylated regions (DMRs) that are associated with ASD, we used the Bioconductor bumphunter package. The bumphunting design matrix accounted for the potential confounding effects of sex, age, batch, and blood cell-type proportions (estimated monocyte, granulocytes, and NK proportions). The analysis identified consecutive CpGs no more than $0.5 \mathrm{~kb}$ apart with an average regional methylation difference $|\Delta \beta|>5 \%$ between cases and controls. Statistical significance was established using 1,000 randomized bootstrap iterations. The resulting DMRs were postfiltered to retain only those with $p$-value $<0.05$ across the DMR and a length (number of consecutive CpGs) of a least three sites. To further enhance stringency, we considered DMRs comprising 
at least one $\mathrm{CpG}$ from the differentially methylated $\mathrm{CpG}$ sites identified between ASD cases and controls, as described above.

\section{Comparisons of Blood Cell Composition in Sample Groups}

As described above, relative proportions of underlying blood cell type were estimated using the Houseman method. We assessed our groups for possible differences in these proportions; the blood cell types measured included, B cell, CD4T, CD8T, monocyte, granulocytes, and NK. These blood cell types were those on which the Houseman method was originally trained, using peripheral blood leukocyte subtypes purchased from AllCells ${ }^{\circledR}$, LLC (Emeryville, CA) or sorted cells from whole blood using negative and positive selection of surface antibodies (Blymphocytes: CD19+; CD4 T-lymphocytes: $\mathrm{CD} 3+, \mathrm{CD} 4+$; $\mathrm{CD} 8$ T-lymphocytes: $\mathrm{CD} 3+, \mathrm{CD} 8+$; monocyte: $\mathrm{CD} 14+$; granulocytes: CD15+; natural killer: $\mathrm{CD} 56+)$ (40). We also calculated the granulocyte/lymphocyte ratio (G/L ratio), which is a common indicator of inflammatory response. As well, the relationship between age and cell type proportion and $\mathrm{G} / \mathrm{L}$ ratio was evaluated in the sample groups using the Spearman's rank correlation coefficient $(r)$ and $p$-value $<0.05$.

\section{Identification of Genetic Variants Associated With ASD}

In our cohorts, we interrogated genetic variation at 366 candidate genes, selected based on the reported SFARI Gene association with ASD. These candidate genes were classified as "Category 1" (high confidence) and "Category 2" (strong candidate) by SFARI Gene at the time of manuscript submission. All the genetic variants were obtained through whole-exome and wholegenome sequencing data using the Genotypes and Phenotypes in Families (GPF) tool (https://gpf.sfari.org/) and MSSNG database (http://www.mss.ng/researchers) for individuals who underwent genome sequencing for investigation of ASD. For each gene, the mode of inheritance was specified, and rare non-synonymous variants were prioritized. For these genes, only de novo variants considered as likely pathogenic or pathogenic with minor allele frequency $<1 \%$ were retained as advised by the guidelines from the American College of Medical Genetics-Association for Molecular Pathology (ACMG-AMP). We then evaluated whether pathogenic de novo variants within ASD risk genes differed in frequency between the ASD subset and the remaining ASD cases.

To further investigate genetic factors involved in ASD, we assessed variants genome-wide (i.e., not limited to SFARI genes), including single nucleotide variants (SNVs), indels, and copy number variants (CNVs), in individuals with ASD from MSSNG $(n=203)$ and SSC $(n=39)$.

Individuals in MSSNG were sequenced using either Complete Genomics or Illumina (HiSeq 2000 or HiSeq X) platforms. SNVs, indels, and CNVs were detected from Complete Genomics samples as previously described (5), and the variants were lifted over from hg19 coordinates to hg38 coordinates for further analysis. For individuals sequenced on Illumina platforms, read alignment (hg38) and SNV/indel detection were performed using the Sentieon pipeline (43). Individuals in SSC were sequenced
TABLE 2 | Clinical measures analyzed in our ASD cohort.

\begin{tabular}{|c|c|c|}
\hline Scale & Subscale & The age range analyzed \\
\hline $\begin{array}{l}\text { WASI, WASI II, WISC IV or } \\
\text { SB-5 }\end{array}$ & $\begin{array}{l}\text { Full Scale Intelligence } \\
\text { Quotient }\end{array}$ & $\begin{array}{l}6-18 \text { years } \\
1-6 \text { years }\end{array}$ \\
\hline \multirow[t]{2}{*}{ ADI-R } & $\begin{array}{l}\text { Communication } \\
\text { domain verbal total }\end{array}$ & $2-18$ years \\
\hline & $\begin{array}{l}\text { Algorithm total scores } \\
\text { [in three domains: } \\
\text { social interaction, } \\
\text { communication, and } \\
\text { restricted repetitive } \\
\text { behavior (RRB)] }\end{array}$ & $2-18$ years \\
\hline \multirow[t]{2}{*}{ ADOS } & $\begin{array}{l}\text { Communication + } \\
\text { Social Interaction } \\
\text { total score }\end{array}$ & $2-18$ years \\
\hline & $\begin{array}{l}\text { Social Affect }+ \\
\text { Restricted Repetitive } \\
\text { Behaviors total score }\end{array}$ & $2-18$ years \\
\hline VABS-II & $\begin{array}{l}\text { Communication } \\
\text { Standard Score }\end{array}$ & $1-6$ years \\
\hline
\end{tabular}

The age range represents the group of ages administered to each clinical measure. WAS, Wechsler Abbreviated Scale of Intelligence; WISC, Wechsler Intelligence Scale for Children; SB, Stanford Binet Intelligence Scales.

on the Illumina HiSeq X platform. SNVs and indels were downloaded from SFARI Base (https://www.sfari.org/resource/ sfari-base). SNVs and indels from both cohorts were annotated using a custom ANNOVAR-based pipeline (44). CNVs were detected in both MSSNG (Illumina samples only) and SSC using a previously-described workflow (45) involving the algorithms ERDS (46) and CNVnator (47). High-confidence de novo SNVs and indels were detected using DeNovoGear (48) as previously described (5). De novo CNVs were identified as those that were detected by both ERDS and CNVnator, that were rare $[<1 \%$ frequency in MSSNG parents and 1,000 Genomes Project (49) population controls], and that were not detected by ERDS or $\mathrm{CNVnator}$ in either parent.

We compared our ASD groups for possible differences in the number of de novo SNVs/indels per individual, and frequency or type of variants (for SNVs and indels: stop gain/ splice site/ frameshift; for CNVs: deletion/duplication).

\section{Examination of Risk Factors and Clinical Phenotypes}

See Table 2 for descriptions of all clinical measures.

We assessed risk factors previously shown to be associated with an increased risk of ASD. These included assisted reproductive technology (ART), gestational age, maternal smoking, parental age at the time of birth, and proband sex. Clinical phenotypes were measured by the following scales: Vineland Adaptive Behavior Scales (VABS-II); ADIR and ADOS as indicators of ASD severity and symptomatology. IQ was assessed using the appropriate scale as determined by the child's age: the Wechsler Abbreviated Scale of Intelligence (WASI, WASI-II), the Wechsler Intelligence Scale of Children-IV (WISC-IV), or the Stanford Binet Intelligence Scales, 5th ed (SB5) (FSIQ score used for each test). These measures, risk factors 
A

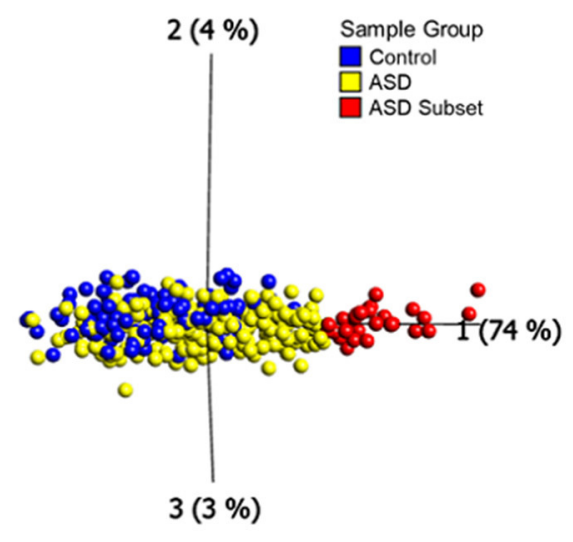

B

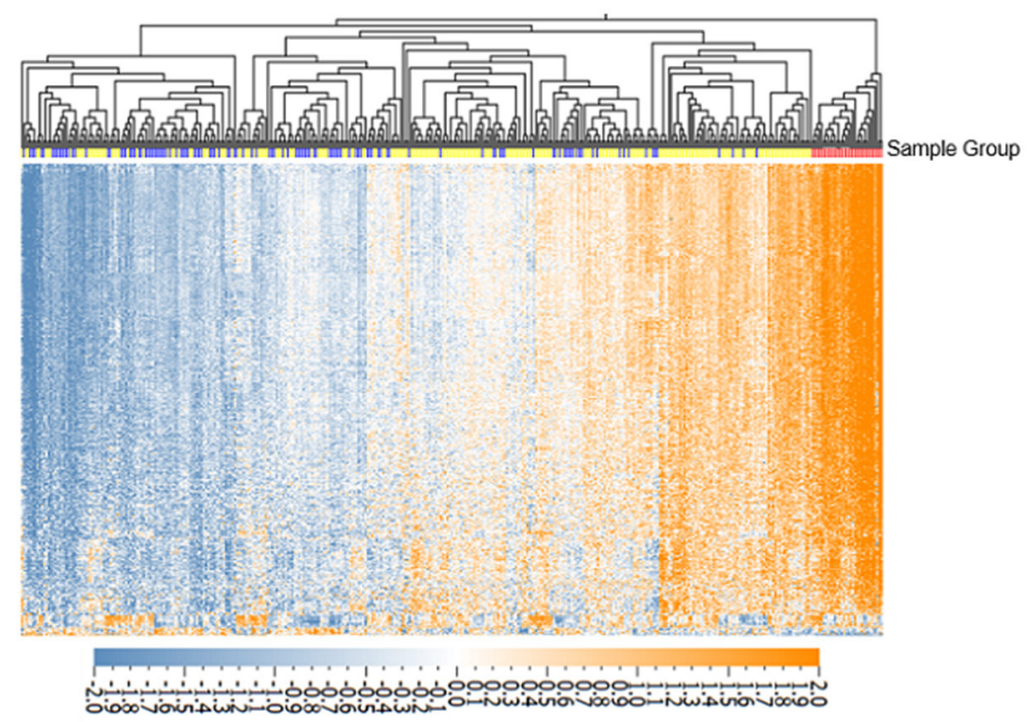

FIGURE 1 | Differential DNAm at 400 in ASD $(n=265)$ and neurotypical controls $(n=122)$ reveals an epigenetically unique subset of ASD cases $(n=32)$. (A) Principal component analysis performed on $400 \mathrm{CpGs}$ (FDR adjusted $p$-value $<0.01$ and $|\Delta \beta|>5 \%$ ), with axes representing first three principal components. (B) Corresponding heatmap hierarchical clustering using Eucledian distance metrics. Orange indicates high DNAm, and blue gray indicates low DNAm, normalized for visualization (mean $=0$, variance $=1$ ). Samples labeled with red and yellow represent the ASD subset and the remaining ASD cases, respectively, blue samples represent controls.

and clinical data were assessed against DNAm in all samples for which they were available, namely ASD cases recruited from the POND network, MSSNG and SSC. Of note, these clinical features were measured in the same study visit as tissue sample collection, or within a 7-month period.

\section{RESULTS}

\section{Identification of DNAm Alterations Associated With ASD}

To investigate DNAm alterations associated with ASD, we compared genome-wide DNAm in blood from individuals with heterogeneous ASD $(n=265)$ to sex- and age-matched, neurotypical controls $(n=122)$ at $427,137 \mathrm{CpG}$ sites (Table 1). We identified 400 significantly differentially methylated CpG sites across the genome at an FDR adjusted $p$-value $<0.01$ and $|\Delta \beta|>5 \%$ (5\% methylation difference) as illustrated by a volcano plot in Supplementary Figure 1 and Supplementary Table 2. Ninety seven percentage of these CpG sites exhibited higher methylation levels (with DNAm differences ranging from 5 to $14 \%$ ) in ASD cases as compared to controls. Principal component analysis (PCA) run on the 400 differentially methylated sites showed a gradient of DNAm values starting from a group of mostly controls to a separate subset of ASD-affected individuals that was epigenetically distinct from both controls and remaining ASD cases ( $n=32$; Figure 1; Table 1). This observation was supported by hierarchical clustering of DNAm values, with this DNAm gradient going from a cluster comprised of mostly controls where the majority of differentially methylated CpGs are hypomethylated to the distinct ASD subset where the majority of these sites are hypermethylated. No significant differences were found between the ASD subset and the remaining ASD cases in terms of age and sex (Mann-Whitney $p$-values $>0.05$ ); both groups of ASD, the subset and remaining cases, included individuals from all three cohorts (POND, MSSNG and SSC; see Table 1 and "Research participants" subsection of Methods for details). Moreover, running an additional limma regression model with DNA collection site included as an additional covariate did not alter the results; of the 400 significantly differentially methylated sites, $381 \mathrm{CpGs}$ remained significant and could still differentiate the ASD subset from the remaining ASD cases (results not shown).

The majority of the differentially methylated CpG sites mapped to promoter regions or gene bodies of 159 RefSeq genes (Supplementary Table 2). The genomic distribution of the 400 CpGs was compared with that of the background test sites. We found a significantly higher proportion of $\mathrm{CpGs}$ located in open sea (72 vs. $35 \%$; $p$-value $<0.05$ ) and a depletion of island CpGs ( 0 vs. $32 \%$; $p<0.01$ ) (Figure $2 \mathrm{~A}$ ). Moreover, the differentially methylated sites were found to be enriched in enhancers, as compared to the full $427,137 \mathrm{CpG}$ sites (49 vs. $23 \%$; $p$-value $<0.05$ ) (Figure 2B).

\section{Functional Enrichment of Differentially Methylated CpG Sites}

We performed gene ontology analysis of the 400 differentially methylated sites using GREAT 4.0.4 to assess enrichment of common biological processes, molecular functions, 
A Differentially methylated CpGs

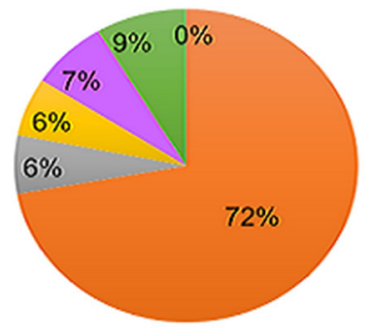

B Differentially methylated CpGs

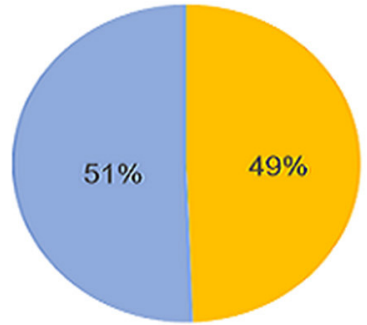

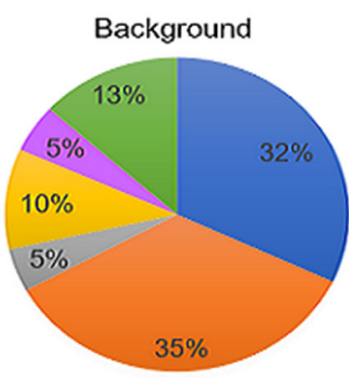

$$
\begin{aligned}
& \text { घsland } \\
& \text { घOpenSea } \\
& \because \text { S_Shelf } \\
& \because \text { S_Shore } \\
& \square \text { N_Shelf } \\
& \square \text { N_Shore }
\end{aligned}
$$

\section{Enhancer}

ves

No

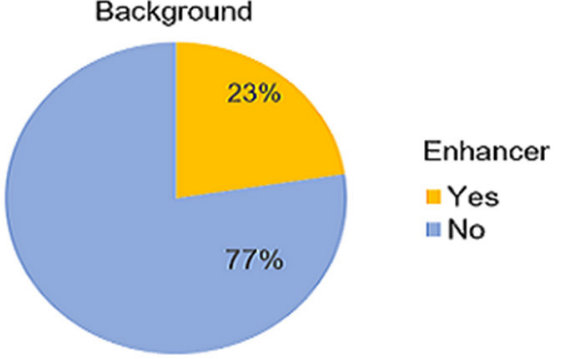

FIGURE 2 | The genomic distribution of the 400 differentially methylated CpG sites identified between ASD cases ( $n=265)$ and controls $(n=122$, left) compared to the background set of all probes that retained after probe filtering $(n=427,137)$ (right). (A) proportion CpG sites in relation to CpG islands and (B) proportion of CpGs overlapping enhancer regions. The differentially methylated sites were found to be significantly enriched in open sea and enhancers ( $p$-values < 0.05$)$ and depleted in CpG islands ( $p$-value < 0.01). "Island" is CpG island; N_shore and S_shore are north (upstream) and south (downstream) shores, i.e. 2kb regions flanking island; N_shelf and S_shelf are north (upstream) and south (downstream) shelves, i.e. 2kb regions flanking island shores.

and cellular components of genes mapping to these $\mathrm{CpG}$ sites. GREAT identified 159 genes that overlapped the 400 CpG sites detected in our differential methylation analysis. We identified 27 GO biological processes assigned to the differentially methylated sites; the majority of them were related to immune function, such as immune and inflammatory response, in addition to enrichment for cellular secretion, as the top GO terms (hypergeometric FDR $q$-value $<0.01$; Supplementary Table 3). Two GO cellular components met significance: granule membrane and inflammasome complex (Supplementary Table 4), while no molecular functions were significantly enriched.

Of the genes mapping to sites of differential methylation ( $n=159$ ), 26 are listed by SFARI as ASD-risk genes (Supplementary Table 5). These included genes encoding proteins of the immune and nervous systems, such as Cullin 3 (CUL3 [MIM: 614496]), ANNEXIN A1 (ANXA1 [MIM:151690]), SH3 and multiple ankyrin repeat domains 2 (SHANK2 [MIM: 613436]) and MET protooncogene (MET [MIM: 164860]).

\section{Identification of Differentially Methylated Regions}

In addition to assessing each $\mathrm{CpG}$ independently, DMRs were evaluated, identifying regional DNAm differences. Significant DMRs were defined by $p<0.05,|\Delta \beta| \geq 5 \%$ and a length of at least three consecutive CpG sites, of which at least one had already been identified as a significant site in our differential methylation analysis (400 sites). Regional DNAm analysis identified 15 significant DMRs (Supplementary Table 6). As expected, these CpGs did not include those that mapped to open sea but rather higher density $\mathrm{CpG}$ regions including island shelves and shores. The longest DMR spanned $0.67 \mathrm{~Kb}$ and mapped to GalactosideBinding, Soluble, 1 (LGALS1) which encodes Galectin-1 involved in regulating apoptosis, cell proliferation and cell differentiation (Supplementary Table 6).

\section{Assessment of Blood Cell Type Composition in DNAm-Based Sample Groups}

To investigate the relationship between the identified enrichment in immune function and inflammatory response associated with the ASD subset signature, we evaluated possible differences in estimated immune blood cell proportions between the three groups: the epigenetically unique ASD subset, the remaining ASD cases, and controls. Interestingly, we identified shifts in cell type proportions in the ASD subset as compared to the remaining ASD cases and controls (Figures 3, 4). Namely, these individuals exhibited a significant increase in CD4T proportion ( $p$-value $<$ $0.01)$. In contrast, granulocyte proportion significantly decreased and accordingly, the G/L ratio was lower $(p$-value $<0.0001)$ in the ASD subset. We found significant differences in blood cell type composition between the remaining ASD cases and controls (Table 3). In addition, we ran 20 iterations of Mann-Whitney tests for each cell type on randomly sampled groups of $n=$ 32 ASD cases (from the ASD cases excluding the DNAm-based subgroup; $n=233$ ) vs. the remaining ASD cases, to ensure the 

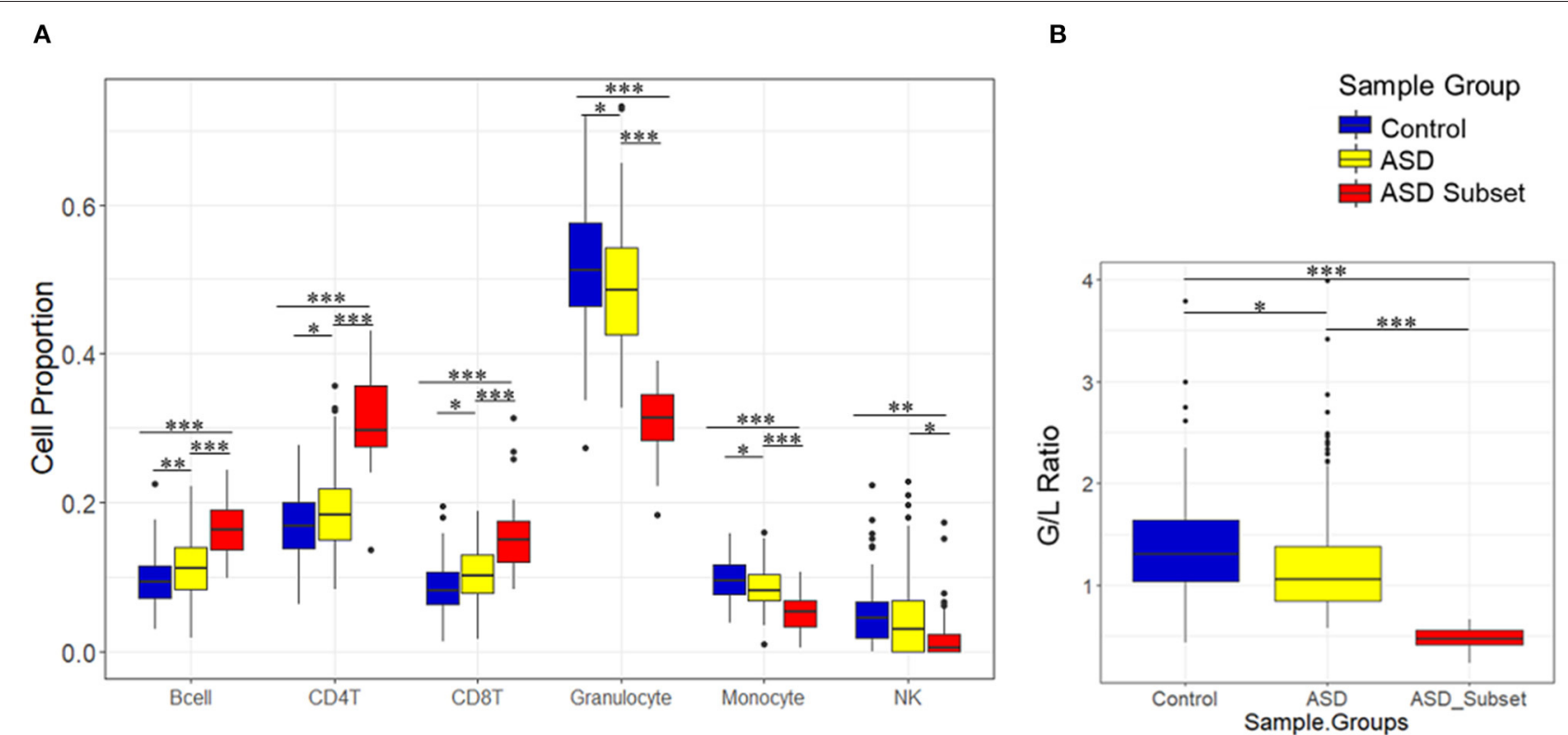

FIGURE 3 | Relative proportions of blood cell types in sample groups, as estimated by DNAm. Boxplots show immune blood cell proportions estimated by the Houseman method $\mathbf{( A )}$ and calculated granulocyte/lymphocyte $(\mathrm{G} / \mathrm{L}$ ) ratio (B). Epigenetically unique ASD subset (red; $n=32$ ), the remaining $\mathrm{ASD}$ cases (yellow; $n=$ 233), and controls (blue; $n=122$ ). ASD subset exhibited significant shifts in cell type proportions and the $\mathrm{G} / \mathrm{L}$ ratio ( $p$-value $<0.01)$ as compared to the remaining ASD cases and controls. Black bars with asterisk represent significant differences in estimated blood cell proportions between the groups $\left({ }^{\star} p \leq 0.05 ;{ }^{* \star} p \leq 0.01 ;{ }^{* \star \star} p\right.$ $\leq 0.001)$

true differences held up to permutation testing. Only a single iteration produced a $p$-value $<0.05$ (seen in monocytes) and no permutation analysis ed $p$-values approached neared those of the true associations (all $p$-values $>0.04$; Supplementary Figure 2).

To investigate if the cell-type proportions was correlated with the age of the samples, we performed correlation analysis based on Pearson correlation coefficient $(r)$ and a $p$-value $<0.05$. We found that in all the ASD cases, age was positively correlated with granulocyte proportion (ASD subset: $r=0.43$, $p$-value $=0.01$; the remaining ASD: $r=0.35$, $p$-value $<0.001$ ) (Figure 5A) and G/L ratio (ASD subset: $r=0.45, p$-value $=0.01$; the remaining ASD: $r=0.37, p$-value $<0.001$ ) (Figure 5B). A significant negative correlation was found between age and CD4T proportion in the ASD cases (ASD subset: $r=-0.4, p$-value $=0.02$; the remaining ASD: $r=-0.2, p$-value $=0.002)$ (Figure 5C). In contrast, the cell type proportions, and the G/L ratio showed no correlation with age in control subjects $(r<0.2, p$-value $>0.5)$.

\section{Reassessment of Differential Methylation Associated With ASD After Removing the ASD Subset}

To further investigate DNAm in our ASD cohort, we removed from our dataset the 32 ASD cases that were detected as unique both epigenetically and in blood cell composition and performed differential methylation analysis between the remaining ASD cases $(n=233)$ and controls $(n=122)$ using the same analytical methods, i.e., limma regression, covarying for age, sex, and estimated cell type proportion (granulocyte, NK, and monocyte). Linear regression analyses identified 77 significantly differentially methylated $\mathrm{CpG}$ sites with FDR adjusted $p$-value $<0.01$ and $|\Delta \beta|>5 \%$ (Supplementary Table 7). Notably, PCA continued to show a gradient of DNAm value tightly associated with granulocytes proportion and the $\mathrm{G} / \mathrm{L}$ ratio across the ASD cases on PC1 (Supplementary Figures 3, 4). As such, epigenetic differences between ASD cases and controls may be attributed either to underlying differences in blood cell composition or the identification of CpG sites that are blood cell type specific.

\section{Identification of Genetic Variants Associated With ASD}

We investigated 366 genes classified by SFARI Gene as high risk for $\mathrm{ASD}$ and looked for differences in pathogenic de novo variant frequency between our DNAm-based ASD groups. In the ASD subset, we identified six individuals (18\%) with nine different de novo pathogenic variants at different genes (Supplementary Table 8). Only 34 individuals (14\%) of the remaining ASD cases harbored de novo pathogenic variants (Supplementary Table 9); most of these were missense variants. Given that the ASD subset did not differ from the rest of the ASD cases in the frequency or type of high-risk variants, we, therefore, expanded our analysis to identify the genome-wide de novo pathogenic variants including SNVs, indels, and CNVs associated with ASD.

Likewise, no significant differences were detected between these two groups of ASD for the average number of de novo $\mathrm{SNV}$ s/indels per individual (average of 1.2 per individual in both groups), and frequency or type of SNVs and indels. However, in the larger ASD cohort, i.e., not the subset with unique DNAm, we 


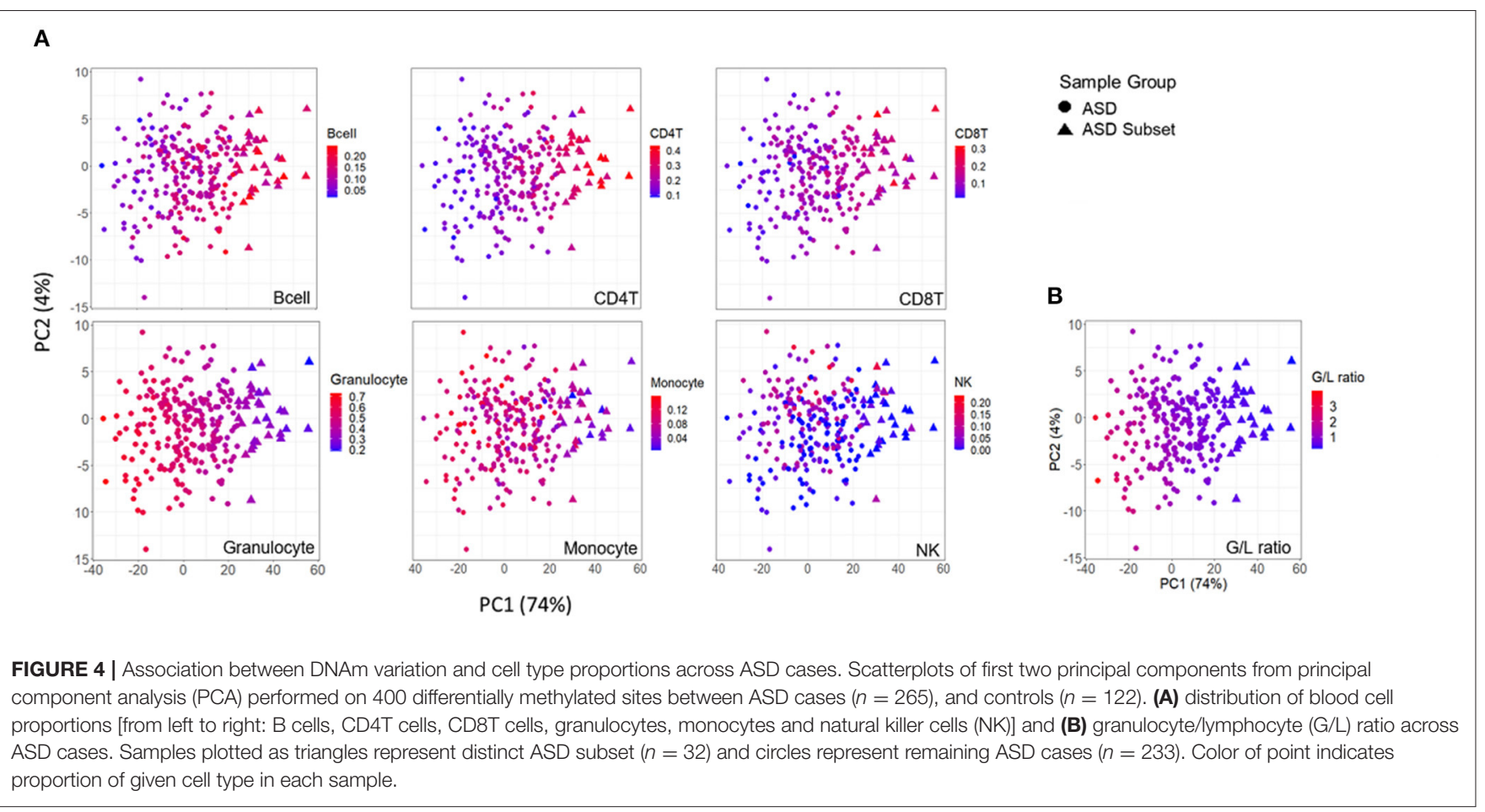

identified nine overlapping $\mathrm{CNV}$ regions of mostly duplications that were identified in more than one patient; The only common deletion identified was assigned to the gene Patched domaincontaining protein 1 (PTCHD1 [MIM: 300828]), which is a high -risk ASD gene (Supplementary Table 10).

\section{Examination of Risk Factors and Clinical Phenotypes in DNAm-Based Groups}

We evaluated the factors that increase the risk of ASD and behavioral phenotypes, comparing the epigenetically unique ASD subset to the remaining ASD cases. No significant differences were detected between these two groups of ASD cases for ART, gestational age, maternal smoking, parental age, and proband sex ( $t$-test $p$-values $>0.05)$. Similarly, clinical phenotypes measured in the cohort did not differ significantly between individuals of the two groups of ASD. Namely, no significant differences were detected between these two groups of ASD cases for ADI-R (Communication domain verbal and algorithm total), ADOS (Communication + social interaction and social Affect + restricted repetitive behaviors) and VABS-II (Communication Standard), and IQ scores (FSIQ) (Table 1).

\section{DISCUSSION}

In this study, we sought to assess genome-wide DNAm alterations associated with ASD. We identified a subset of ASD cases that exhibit differential methylation patterns distinct from both controls and the remaining ASD group as well as significant shifts in cell type proportions, i.e., the granulocyte-to-lymphocyte ratio was significantly lower in the ASD subset than in the remaining ASD cases and controls. In the present study, beyond blood cell composition, we found no significant differences between the ASD subset and the remaining ASD cohort, including sex, age, genetic risk variants or clinical measures such as ADI, VABS and ADOS subscale scores. Furthermore, our study provides additional support for previously reported involvement of SHANK2, ANXA1, MET, CUL3 and other genes in the pathophysiology of ASD. Our study suggests that at least one mechanism underpinning differential methylation between ASD cases and neurotypical controls is a difference in blood cell type proportion.

It is important to note that blood cell type proportion was estimated from DNA methylation. As such, it is possible that differences observed may be attributed to true changes in the blood cell composition or that DNA methylation alterations exist in the ASD subgroup at CpG sites used to estimate blood cell composition. A previous meta-analysis of methylation studies of ASD by Andrews et al. (33) reported in their patient demographics, significant differences in granulocyte and B cell proportions between ASD cases and control subjects parallel to those found in our study. These investigators found no single $\mathrm{CpG}$ to meet genome-wide significance using Bonferroni correction $\left(p<1.12 \times 10^{-7}\right)$ for the association between ASD and DNAm and did not interpret cell types differences in the discussion (33). Regardless, it is plausible that these methylation alterations may be indicative of altered immune function in the ASD subset. As well, this is not the first instance in which DNAm has been used to identify changes in blood cell proportion associated with a disorder; this has been reported in both asthma and systemic lupus erythematosus by Kong et al. (50). They showed that the proportion of DNAm alterations attributable to changes in cell type composition varies considerably in both 


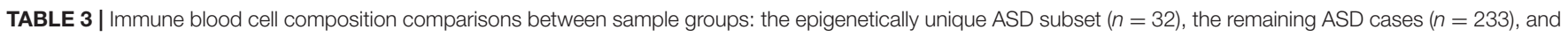
controls $(n=122)$.

\begin{tabular}{|c|c|c|c|}
\hline \multirow[t]{2}{*}{ Cell type composition } & \multicolumn{3}{|c|}{ Comparison groups } \\
\hline & $\begin{array}{l}\text { ASD subset vs. ASD } \\
\text { mean difference } \pm \text { SE }\end{array}$ & $\begin{array}{l}\text { ASD subset vs. Control } \\
\text { mean difference } \pm \text { SE }\end{array}$ & $\begin{array}{c}\text { ASD vs. Control } \\
\text { mean difference } \pm \text { SE }\end{array}$ \\
\hline B cell & $0.05 \pm 0.01^{\star \star \star}$ & $0.07 \pm 0.01^{\star \star \star}$ & $0.02 \pm 0.003^{\star \star}$ \\
\hline CD4T & $0.13 \pm 0.01^{\star \star \star}$ & $0.14 \pm 0.01^{\star \star \star}$ & $0.01 \pm 0.005^{\star}$ \\
\hline CD8T & $0.06 \pm 0.01^{\star \star \star}$ & $0.07 \pm 0.01^{\star \star \star}$ & $0.01 \pm 0.003^{*}$ \\
\hline Granulocytes & $-0.20 \pm 0.01^{\star \star \star}$ & $-0.21 \pm 0.01^{\star \star \star}$ & $-0.01 \pm 0.01^{\star}$ \\
\hline Monocytes & $-0.03 \pm 0.004^{\star \star \star}$ & $-0.04 \pm 0.003^{\star \star \star}$ & $-0.01 \pm 0.002^{\star}$ \\
\hline NK & $-0.02 \pm 0.01^{\star}$ & $-0.03 \pm 0.01^{\star \star}$ & $-0.01 \pm 0.004$ \\
\hline $\mathrm{G} / \mathrm{L}$ ratio & $-0.72 \pm 0.03^{\star \star \star *}$ & $-0.92 \pm 0.05^{\star \star \star}$ & $-0.18 \pm 0.05^{\star}$ \\
\hline
\end{tabular}

NK, Natural killer cell; G/L, Granulocyte/Lymphocyte.

${ }^{*} p \leq 0.05 ;{ }^{* *} p \leq 0.01 ;{ }^{* \star *} p \leq 0.001$.

asthma and systemic lupus erythematosus, suggesting diseasespecific cell subtype proportion changes contributing to DNAm alterations (50). Future studies using differential blood counts and DNAm-based blood cell estimates from the same blood draws are required to clarify this relationship.

Two additional findings in our study support the observed relationship between DNAm, immune cell type and ASD in our cohorts. The majority of the differentially methylated CpG sites were enriched for gene ontology categories implicated in immune and inflammatory response (Supplementary Table 4), which strengthens the scientific evidence that a dysregulated immune system is one of the contributing factors in ASD (51). Our findings are consistent with the methylation analysis in brain that demonstrates altered immune response in the cortical region of autistic cases, Brodmann area 10, correspond with epigenetic modulation of genomic regions relevant to several categories related to immune response, including inflammatory response to antigens and positive regulation of cytokine biosynthetic processes (16). As well, brain and blood transcriptome studies show that many of the genes exhibiting a higher variability in their overall expression pattern were related to the immune system in autistic individuals, indicating dysregulation in immune functions in ASD (52-55). A comparison between our differentially methylated sites and the transcriptomic data reported in gene expression studies showed overlap with two genes characterized by significant DNA hypermethylation in our ASD subset and decreased expression levels. The ANXA1 gene, which encodes a protein that functions in adaptive immunity, was found to be upregulated in ASD individuals (56). As well, ankyrin repeat domain 22 (ANKRD22) overlapping three differentially methylated CpGs was found to be significantly downregulated in blood (57); this gene encodes a protein that specifically interacts with STING, a critical protein function in multiple anti-viral innate immune pathways (58). The prior evidence of hypermethylation of the immune-related genes correlating with decreased expression further supports the potential role of epigenetic regulation of the altered immune response associated with ASD. Further, when the genome-wide linear regression of ASD vs. controls was rerun with the ASD subset removed, the differentially methylated CpG sites were still strongly predictive of blood cell proportion.

We searched for overlap between differentially methylated sites in our blood DNAm and previous studies of DNAm in individuals with ASD. We found 23 overlapping CpG sites with DNAm signature detected in adult cortical regions (16); CpG sites overlapped notable genes such as syndecan-2 (SDC2 [MIM:142460]), Dystonin (DST [MIM: 113810) and mediator complex subunit 12L (MED12L [MIM: 611318]); However, we did not find any overlap with the findings of blood DNAm studies $(31,32)$.

In recent years, there is emerging evidence and growing concern that a dysregulated or abnormal immune response may be involved in the development of some forms of ASD. Several lines of research have provided substantial evidence of immune dysregulation in subsets of individuals with ASD, including skewed inflammation responses, cytokines, and total numbers and frequencies of immune cells (59-61). The inconsistencies in previous research findings are marked by considerable variation in the prevalence of ASD by ethnicity/race, sex, geographic area, and level of intellectual ability. The heterogeneity of ASD is the source of much difficulty in study underlying pathophysiology, etiology and biomarkers of this neurodevelopmental disorder. This is especially apparent in genetic studies of ASD, in which rare SNVs and CNVs account for only a small proportion of ASD risk. Biological measures, such as DNAm or immune markers, which exhibit consistent changes across substantial subsets of individuals with ASD, may provide a new avenue for ASD research.

A number of limitations should be noted. As described above, cell types composition was not measured directly but rather estimated using specific $\mathrm{CpG}$ sites that exhibit blood cell-type specific DNAm patterns. While this method has been validated and is widely used, it is possible that DNAm levels at these CpGs sites were altered by factors other than cell type, causing skewed estimates. However, it is worth noting that cell type of origin is one of the strongest predictors of DNAm patterns. For example, DNAm patterns from a single tissue sampled from two individuals are more strongly 

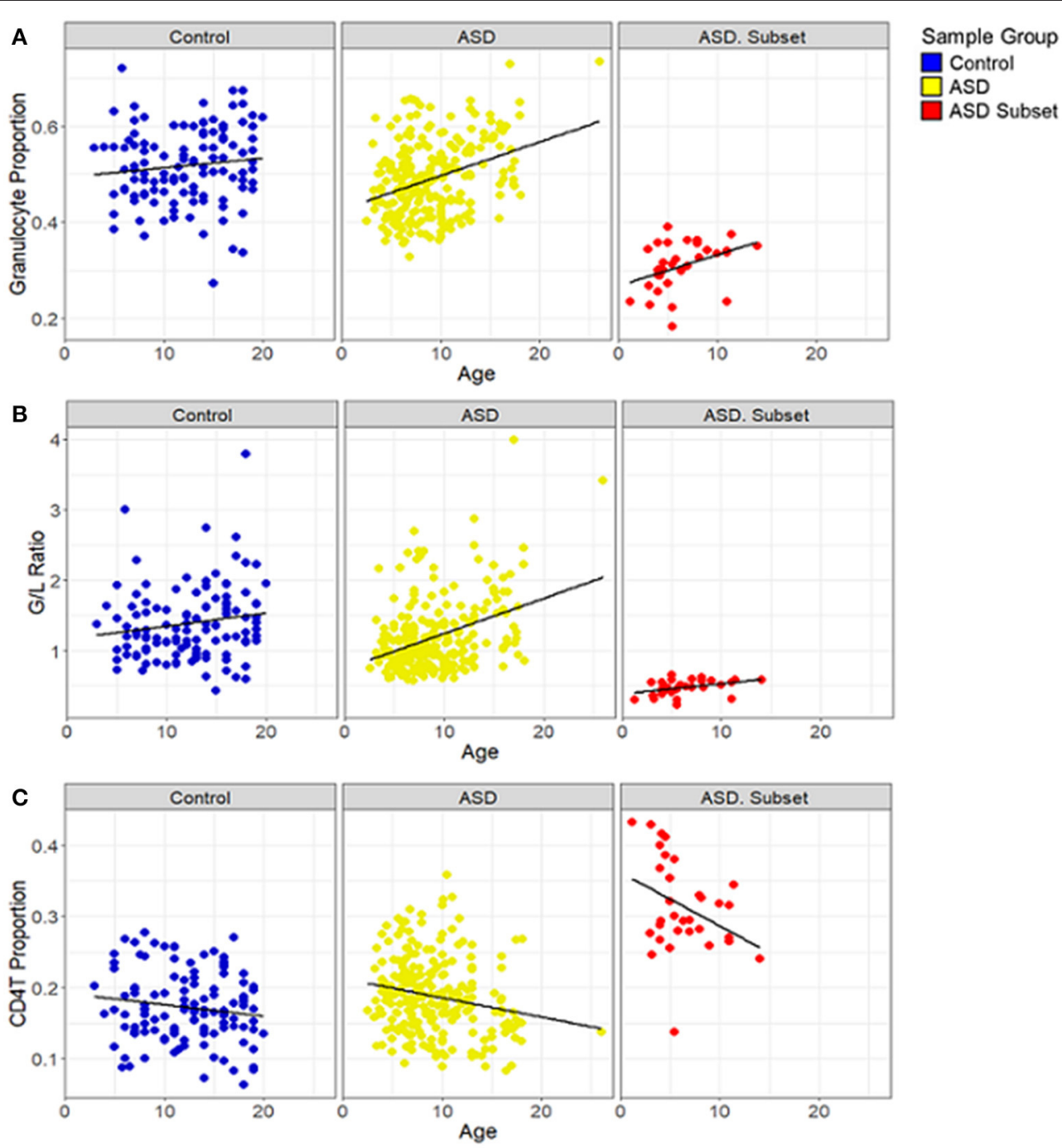

FIGURE 5 | Relationship between blood cell proportions and sample age in individuals with ASD. Box plots depict (A) granulocyte proportion, (B) granulocyte/lymphocyte (G/L) ratio and (C) CD4T proportion in samples plotted against age. ASD subset (red; $n=32$ ), the remaining ASD cases (yellow; $n=233$ ), and controls (blue; $n=122$ ). In all both ASD groups, age was positively correlated with the granulocyte proportion (ASD subset: $r=0.43, p$-value $=0.01$; the remaining ASD: $r=0.35, p$-value < 0.001) and the G/L ratio (ASD subset: $r=0.45, p$-value $=0.01$; the remaining ASD: $r=0.37, p$-value $<0.001$ ) and negatively correlated with CD4T (ASD subset: $r=-0.4, p$-value $=0.02$; the remaining ASD: $r=-0.2, p$-value $=0.002$ ); the remaining ASD: $r=-0.2, p$-value $=0.002$ ). In controls, no significant correlation was found between age and the blood cell compositions.

correlated than patterns from a single individual in different tissues. Beyond this, risk factors and clinical phenotypes were available for only a subset of ASD individuals in this study, which may considerably reduce the statistical power to detect associations between DNAm and behavioral phenotype. This missing information is also important as it may have contributed to an unbalanced study design. The distribution of our nominal $p$-values suggested genomic inflation (see QQ-plot in Supplementary Figure 5), which is associated with inflated false positives. Although this can be result of an unbalanced study design or confounding factors that are not accounted for in the statistical model, it may also result from a strong association between DNAm and ASD status at a large number of CpGs sites (62). We propose that by testing the effect of systematic blood cell composition differences, as observed between our cohorts, on DNAm changes we expect broad, genome-wide differences with large effect sizes that would mimic genomic inflation. Furthermore, we accounted for important covariates, including technical factors, blood cell proportions, sex, age, etc. and only reported CpGs that met stringent significance threshold of FDR adjusted $p$-value $<0.01$ and $\Delta \beta \mid$ $>0.05$. Nonetheless, this observation which can sometimes reflect genomic inflation does support the need for independent replication of these findings in future to better understand the relationship between ASD, blood cell composition and DNAm levels. 


\section{CONCLUSION}

In summary, this study demonstrates a gradient of DNAm alterations across our ASD cases tightly associated with shifts in immune cell type proportions. Moreover, we report an epigenetically unique subset of ASD cases that exhibited a significant difference in immune cell type proportions, as compared to the controls and the remaining ASD cases. Our findings build on past reports of changes in the immune systems of children with ASD, supporting the potential role of altered immunological mechanisms in the complex pathophysiology of ASD. The discovery of significant molecular and immunological features in subgroups of individuals with ASD provides unique insight into the molecular pathophysiology of ASD that can help clinicians to better stratify patients, facilitating personalized interventions and improved outcomes. These results may lead to the hypothesis that immunological shifts may induce longterm changes through modulation of DNA methylation in genomic regions involved in the immune response, such as the hypermethylated regions observed in the subset of ASD cases in our data.

\section{DATA AVAILABILITY STATEMENT}

The datasets generated for this article are not readily available because the consents obtained did not cover open access/sharing of the data. Requests to access the datasets should be directed to Rosanna Weksberg, rweksb@sickkids.ca.

\section{ETHICS STATEMENT}

The studies involving human participants were reviewed and approved by Research Ethics Board (REB), The Hospital for Sick Children, Toronto, Canada. REB number: 0019980189. Written informed consent to participate in this study was provided by the participants' legal guardian/next of kin.

\section{REFERENCES}

1. Martin G. Diagnostic and statistical manual of mental disorders: DSM-5 (5th edition). Ref Rev. (2014) 28:36-7. doi: 10.1108/RR-10-2013-0256

2. Bridgemohan C, Cochran DM, Howe YJ, Pawlowski K, Zimmerman AW, Anderson GM, et al. Investigating potential biomarkers in autism spectrum disorder. Front Integr Neurosci. (2019) 13:31. doi: 10.3389/fnint.2019.00031

3. Veenstra-VanderWeele J, Blakely RD. Networking in autism: leveraging genetic, biomarker and model system findings in the search for new treatments. Neuropsychopharmacology. (2012) 37:196-212. doi: 10.1038/npp.2011.185

4. Ruggeri B, Sarkans U, Schumann G, Persico AM. Biomarkers in autism spectrum disorder: the old and the new. Psychopharmacology. (2014) 231:1201-16. doi: 10.1007/s00213-013-3290-7

5. C Yuen RK, Merico D, Bookman M, L Howe J, Thiruvahindrapuram B, Patel $\mathrm{RV}$, et al. Whole genome sequencing resource identifies 18 new candidate genes for autism spectrum disorder. Nature neuroscience. (2017) 20:60211. doi: 10.1038/nn.4524

6. Satterstrom FK, Kosmicki JA, Wang J, Breen MS, De Rubeis S, An JY, et al. Large-scale exome sequencing study implicates both developmental and

\section{AUTHOR CONTRIBUTIONS}

MJ and SGo analyzed and interpreted the data and wrote the manuscript. SC participated in study design and interpreted the data. BT generated genomic variant data. SS collected patient samples and performed genome-wide sequencing to identify variants. EK, MA, RN, SGe, JC, RS, SS, and EA are members of the executive committee of the POND Network, which provided patient cohorts and phenotype data. EG assisted with the interpretation of immune cell type data and provided input on study design. RW is the principal investigator and was involved in all aspects of the study. All co-authors edited and revised the manuscript.

\section{FUNDING}

This work was supported by the Ontario Brain Institute. OBI is an independent non-profit corporation, funded partially by the Ontario government. The opinions, results and conclusions are those of the authors and no endorsement by the Ontario Brain Institute is intended or should be inferred.

\section{ACKNOWLEDGMENTS}

We were grateful to all the patients and families who participated in this research and to the many clinicians for their assistance with recruitment. We also would also like to thank Youliang Lou, Chunhua Zhao and Khadine Wiltshire for their invaluable contributions to this work.

\section{SUPPLEMENTARY MATERIAL}

The Supplementary Material for this article can be found online at: https://www.frontiersin.org/articles/10.3389/fneur. 2021.612817/full\#supplementary-material

functional changes in the neurobiology of autism. Cell. (2020) 180:56884.e23. doi: 10.1016/j.cell.2019.12.036

7. Grove J, Ripke S, Als TD, Mattheisen M, Walters RK, Won H, et al. Identification of common genetic risk variants for autism spectrum disorder. Nat Genet. (2019) 51:431-44. doi: 10.1038/s41588-019-0344-8

8. Carter MT, Scherer SW. Autism spectrum disorder in the genetics clinic: a review. Clin Genet. (2013) 83:399-407. doi: 10.1111/cge.12101

9. Huguet G, Ey E, Bourgeron T. The genetic landscapes of autism spectrum disorders. Annu Rev Genomics Hum Genet. (2013) 14:191213. doi: 10.1146/annurev-genom-091212-153431

10. Tammimies K, Marshall CR, Walker S, Kaur G, Thiruvahindrapuram B, Lionel AC, et al. Molecular diagnostic yield of chromosomal microarray analysis and whole-exome sequencing in children with autism spectrum disorder. JAMA. (2015) 314:895-903. doi: 10.1001/jama.2015.10078

11. Almandil NB, Alkuroud DN, AbdulAzeez S, AlSulaiman A, Elaissari A, Borgio JF. Environmental and genetic factors in autism spectrum disorders: special emphasis on data from arabian studies. Int J Environ Res Public Health. (2019) 16:658. doi: 10.3390/ijerph16040658

12. Nestadt G, Grados M, Samuels JF. Genetics of obsessive-compulsive disorder. Psychiatr Clin North Am. (2010) 33:141-58. doi: 10.1016/j.psc.2009.11.001 
13. Skinner MK. Role of epigenetics in developmental biology and transgenerational inheritance. Birth Defects Res C Embryo Today. (2011) 93:51-5. doi: 10.1002/bdrc.20199

14. Grafodatskaya D, Chung B, Szatmari P, Weksberg R. Autism spectrum disorders and epigenetics. Autism Epigenetics. (2010) 49:794-809. doi: 10.1016/j.jaac.2010.05.005

15. Zhu L, Wang $\mathrm{X}$, Li XL, Towers A, Cao X, Wang $\mathrm{P}$, et al. Epigenetic dysregulation of SHANK3 in brain tissues from individuals with autism spectrum disorders. Hum Mol Genet. (2014) 23:1563-78. doi: 10.1093/hmg/ddt547

16. Nardone S, Sams DS, Reuveni E, Getselter D, Oron O, Karpuj M, et al. DNA methylation analysis of the autistic brain reveals multiple dysregulated biological pathways. Transl Psychiatry. (2014) 4:e433. doi: 10.1038/tp.2014.70

17. Gregory SG, Connelly JJ, Towers AJ, Johnson J, Biscocho D, Markunas CA, et al. Genomic and epigenetic evidence for oxytocin receptor deficiency in autism. BMC Med. (2009) 7:62. doi: 10.1186/1741-7015-7-62

18. Nagarajan RP, Hogart AR, Gwye Y, Martin MR, LaSalle JM. Reduced MeCP2 expression is frequent in autism frontal cortex and correlates with aberrant MECP2 promoter methylation. Epigenetics. (2006) 1:e111. doi: 10.4161/epi.1.4.3514

19. Lasalle JM. Autism genes keep turning up chromatin. OA Autism. (2013) 1:14. doi: 10.13172/2052-7810-1-2-610

20. Pinto D, Delaby E, Merico D, Barbosa M, Merikangas A, Klei L, et al. Convergence of genes and cellular pathways dysregulated in autism spectrum disorders. Am J Hum Genet. (2014) 94:677-94. doi: 10.1016/j.ajhg.2014.03.018

21. Butcher DT, Cytrynbaum C, Turinsky AL, Siu MT, Inbar-Feigenberg M, Mendoza-Londono R, et al. CHARGE and kabuki syndromes: genespecific DNA methylation signatures identify epigenetic mechanisms linking these clinically overlapping conditions. Am J Hum Genet. (2017) 100:77388. doi: 10.1016/j.ajhg.2017.04.004

22. Iwase S, Bérubé NG, Zhou Z, Kasri NN, Battaglioli E, Scandaglia M, et al. Epigenetic etiology of intellectual disability. J Neurosci Nurs. (2017) 37:1077382. doi: 10.1523/JNEUROSCI.1840-17.2017

23. Wong CC, Smith RG, Hannon E, Ramaswami G, Parikshak NN, Assary A, et al. Genome-wide DNA methylation profiling identifies convergent molecular signatures associated with idiopathic and syndromic autism in post-mortem human brain tissue. Hum Mol Genet. (2019) 28:220111. doi: $10.1093 / \mathrm{hmg} / \mathrm{ddz} 052$

24. Ladd-Acosta C, Hansen KD, Briem E, Fallin MD, Kaufmann WE, Feinberg AP. Common DNA methylation alterations in multiple brain regions in autism. Mol Psychiatry. (2014) 19:862-71. doi: 10.1038/mp.2013.114

25. Zhubi A, Chen Y, Guidotti A, Grayson DR. Epigenetic regulation of RELN and GAD1 in the frontal cortex (FC) of autism spectrum disorder (ASD) subjects. Int J Dev Neurosci. (2017) 62:63-72. doi: 10.1016/j.ijdevneu.2017.02.003

26. James SJ, Shpyleva S, Melnyk S, Pavliv O, Pogribny IP. Complex epigenetic regulation of engrailed-2 (EN-2) homeobox gene in the autism cerebellum. Transl Psychiatry. (2013) 3:e232. doi: 10.1038/tp.2013.8

27. Choufani S, Gibson WT, Turinsky AL, Chung BHY, Wang T, Garg K, et al. DNA methylation signature for EZH2 functionally classifies sequence variants in three PRC2 complex genes. Am J Hum Genet. (2020) 106:596610. doi: 10.1016/j.ajhg.2020.03.008

28. Choufani S, Cytrynbaum C, Chung BH, Turinsky AL, Grafodatskaya D, Chen YA, et al. NSD1 mutations generate a genome-wide DNA methylation signature. Nat Commun. (2015) 6:10207. doi: 10.1038/ncomms10207

29. Aref-Eshghi E, Kerkhof J, Pedro VP, Groupe DIF, Barat-Houari M, RuizPallares N, et al. Evaluation of DNA methylation episignatures for diagnosis and phenotype correlations in 42 mendelian neurodevelopmental disorders. Am J Hum Genet. (2020) 106:356-70. doi: 10.1016/j.ajhg.2020.01.019

30. Siu MT, Butcher DT, Turinsky AL, Cytrynbaum C, Stavropoulos DJ, Walker $\mathrm{S}$, et al. Functional DNA methylation signatures for autism spectrum disorder genomic risk loci: 16p11.2 deletions and CHD8 variants. Clin Epigenetics. (2019) 11:103. doi: 10.1186/s13148-019-0684-3

31. Liang $\mathrm{S}$, Li Z, Wang $\mathrm{Y}, \mathrm{Li} \mathrm{X}$, Yang $\mathrm{X}$, Zhan $\mathrm{X}$, et al. Genome-wide DNA methylation analysis reveals epigenetic pattern of SH2B1 in Chinese monozygotic twins discordant for autism spectrum disorder. Front Neurosci. (2019) 13:712. doi: 10.3389/fnins.2019.00712

32. Wong CC, Meaburn EL, Ronald A, Price TS, Jeffries AR, Schalkwyk LC, et al. Methylomic analysis of monozygotic twins discordant for autism spectrum disorder and related behavioural traits. Mol Psychiatry. (2014) 19:495-503. doi: 10.1038/mp.2013.41

33. Andrews SV, Sheppard B, Windham GC, Schieve LA, Schendel DE, Croen LA, et al. Case-control meta-analysis of blood DNA methylation and autism spectrum disorder. Mol Autism. (2018) 9:40. doi: 10.1186/s13229-018-0224-6

34. Galanter JM, Gignoux CR, Oh SS, Torgerson D, Pino-Yanes M, Thakur $\mathrm{N}$, et al. Differential methylation between ethnic sub-groups reflects the effect of genetic ancestry and environmental exposures. eLife. (2017) 6:6. doi: 10.7554/eLife.20532

35. Lord C, Rutter M, Le Couteur A. Autism Diagnostic Interview-Revised: a revised version of a diagnostic interview for caregivers of individuals with possible pervasive developmental disorders. J Autism Dev Disord. (1994) 24:659-85. doi: 10.1007/BF02172145

36. Lord C, Risi S, Lambrecht L, Cook EH Jr, Leventhal BL, DiLavore PC, et al. The autism diagnostic observation schedule-generic: a standard measure of social and communication deficits associated with the spectrum of autism. $J$ Autism Dev Disord. (2000) 30:205-23. doi: 10.1023/A:1005592401947

37. Lord C, Rutter M, DiLavore PC, Risi S, Gotham K, Bishop SL. Autism Diagnostic Observation Schedule 2nd Edn. (ADOS-2). Torrance, CA: Western Psychological Services (2012).

38. Aryee MJ, Jaffe AE, Corrada-Bravo H, Ladd-Acosta C, Feinberg AP, Hansen KD, et al. Minfi: a flexible and comprehensive Bioconductor package for the analysis of Infinium DNA methylation microarrays. Bioinformatics. (2014) 30:1363-9. doi: 10.1093/bioinformatics/btu049

39. Ritchie ME, Phipson B, Wu D, Hu Y, Law CW, Shi W, et al. limma powers differential expression analyses for RNA-sequencing and microarray studies. Nucleic Acids Res. (2015) 43:e47. doi: 10.1093/nar/gkv007

40. Houseman EA, Accomando WP, Koestler DC, Christensen BC, Marsit CJ, Nelson $\mathrm{HH}$, et al. DNA methylation arrays as surrogate measures of cell mixture distribution. BMC Bioinformatics. (2012) 13:86. doi: 10.1186/1471-2105-13-86

41. Benjamini Y, Hochberg Y. Controlling the false discovery rate: a practical and powerful approach to multiple testing. J R Stat Soc [Ser B]. (1995) 57:289-300. doi: 10.1111/j.2517-6161.1995.tb02031.x

42. McLean CY, Bristor D, Hiller M, Clarke SL, Schaar BT, Lowe CB, et al. GREAT improves functional interpretation of cis-regulatory regions. Nat Biotechnol. (2010) 28:495-501. doi: 10.1038/nbt.1630

43. Kendig KI, Baheti S, Bockol MA, Drucker TM, Hart SN, Heldenbrand JR, et al. Sentieon DNASeq variant calling workflow demonstrates strong computational performance and accuracy. Front Genet. (2019) 10:736. doi: 10.3389/fgene.2019.00736

44. Wang K, Li M, Hakonarson H. ANNOVAR: functional annotation of genetic variants from high-throughput sequencing data. Nucleic Acids Res. (2010) 38:e164. doi: 10.1093/nar/gkq603

45. Trost B, Walker S, Wang Z, Thiruvahindrapuram B, MacDonald JR, Sung WWL, et al. A comprehensive workflow for read depth-based identification of copy-number variation from whole-genome sequence data. Am J Hum Genet. (2018) 102:142-55. doi: 10.1016/j.ajhg.2017.12.007

46. Zhu M, Need AC, Han Y, Ge D, Maia JM, Zhu Q, et al. Using ERDS to infer copy-number variants in high-coverage genomes. Am J Hum Genet. (2012) 91:408-21. doi: 10.1016/j.ajhg.2012.07.004

47. Abyzov A, Urban AE, Snyder M, Gerstein M. CNVnator: an approach to discover, genotype, and characterize typical and atypical CNVs from family and population genome sequencing. Genome Res. (2011) 21:97484. doi: $10.1101 /$ gr.114876.110

48. Ramu A, Noordam MJ, Schwartz RS, Wuster A, Hurles ME, Cartwright RA, et al. DeNovoGear: de novo indel and point mutation discovery and phasing. Nat Methods. (2013) 10:985-7. doi: 10.1038/nmeth.2611

49. Genomes Project C, Auton A, Brooks LD, Durbin RM, Garrison EP, Kang $\mathrm{HM}$, et al. A global reference for human genetic variation. Nature. (2015) 526:68-74. doi: 10.1038/nature15393

50. Kong Y, Rastogi D, Seoighe C, Greally JM, Suzuki M. Insights from deconvolution of cell subtype proportions enhance the interpretation of functional genomic data. PLoS ONE. (2019) 14:e0215987. doi: 10.1371/journal.pone.0215987

51. Depino AM. Peripheral and central inflammation in autism spectrum disorders. Mol Cell Neurosci. (2013) 53:69-76. doi: 10.1016/j.mcn.2012. 10.003 
52. Voineagu I, Wang X, Johnston P, Lowe JK, Tian Y, Horvath S, et al. Transcriptomic analysis of autistic brain reveals convergent molecular pathology. Nature. (2011) 474:380-4. doi: 10.1038/ nature 10110

53. Garbett K, Ebert PJ, Mitchell A, Lintas C, Manzi B, Mirnics K, et al. Immune transcriptome alterations in the temporal cortex of subjects with autism. Neurobiol Dis. (2008) 30:303-11. doi: 10.1016/j.nbd.2008.01.012

54. Saffari A, Arno M, Nasser E, Ronald A, Wong CCY, Schalkwyk LC, et al. RNA sequencing of identical twins discordant for autism reveals bloodbased signatures implicating immune and transcriptional dysregulation. $\mathrm{Mol}$ Autism. (2019) 10:38. doi: 10.1186/s13229-019-0285-1

55. Filosi M, Kam-Thong T, Essioux L, Muglia P, Trabetti E, Spooren W, et al. Transcriptome signatures from discordant sibling pairs reveal changes in peripheral blood immune cell composition in Autism Spectrum Disorder. Transl Psychiatry. (2020) 10:106. doi: 10.1038/s41398-020-0778-x

56. Chien WH, Gau SS, Chen $\mathrm{CH}$, Tsai WC, Wu YY, Chen PH, et al. Increased gene expression of FOXP1 in patients with autism spectrum disorders. $\mathrm{Mol}$ Autism. (2013) 4:23. doi: 10.1186/2040-2392-4-23

57. Glatt SJ, Tsuang MT, Winn M, Chandler SD, Collins M, Lopez L, et al. Blood-based gene expression signatures of autistic infants and toddlers. J Am Acad Child Adolesc Psychiatry. (2012) 51:934-44.e2. doi: 10.1016/j.jaac.2012. 07.007

58. Bin L, Li X, Richers B, Streib JE, Hu JW, Taylor P, et al. Ankyrin repeat domain 1 regulates innate immune responses against herpes simplex virus 1: a potential role in eczema herpeticum. J Allergy Clin Immunol. (2018) 141:2085-93.e1. doi: 10.1016/j.jaci.2018.01.001
59. Ashwood P, Wills S, Van de Water J. The immune response in autism: a new frontier for autism research. J Leukoc Biol. (2006) 80:115. doi: 10.1189/jlb.1205707

60. Ashwood P, Krakowiak P, Hertz-Picciotto I, Hansen R, Pessah I, Van de Water J. Elevated plasma cytokines in autism spectrum disorders provide evidence of immune dysfunction and are associated with impaired behavioral outcome. Brain Behav Immun. (2011) 25:40-5. doi: 10.1016/j.bbi.2010.08.003

61. Hughes HK, Mills Ko E, Rose D, Ashwood P. Immune dysfunction and autoimmunity as pathological mechanisms in autism spectrum disorders. Front Cell Neurosci. (2018) 12:405. doi: 10.3389/fncel.2018.00405

62. Yang J, Weedon MN, Purcell S, Lettre G, Estrada K, Willer CJ, et al. Genomic inflation factors under polygenic inheritance. Eur J Hum Genet. (2011) 19:807-12. doi: 10.1038/ejhg.2011.39

Conflict of Interest: The authors declare that the research was conducted in the absence of any commercial or financial relationships that could be construed as a potential conflict of interest.

Copyright (C) 2021 Jangjoo, Goodman, Choufani, Trost, Scherer, Kelley, Ayub, Nicolson, Georgiades, Crosbie, Schachar, Anagnostou, Grunebaum and Weksberg. This is an open-access article distributed under the terms of the Creative Commons Attribution License (CC BY). The use, distribution or reproduction in other forums is permitted, provided the original author(s) and the copyright owner(s) are credited and that the original publication in this journal is cited, in accordance with accepted academic practice. No use, distribution or reproduction is permitted which does not comply with these terms. 